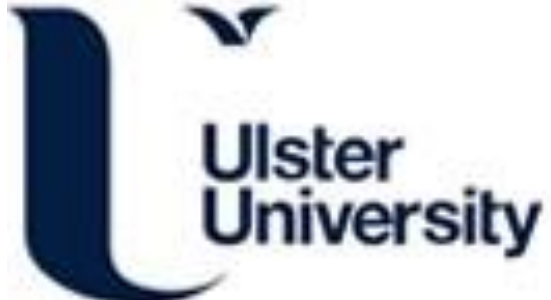

\section{A Holistic Technology-Based Solution for Prevention and Management of Diabetic Foot Complications}

Cleland, I., Rafferty, J., Synnott, J., Cundell, J., Boyd, A., Nugent, C., Chaurasia, P., Morrison, G., Madill, G., Burns, S., \& Monk- Ozgul, L. (2017). A Holistic Technology-Based Solution for Prevention and Management of Diabetic Foot Complications. In Unknown Host Publication (pp. 453-465). Springer.

Link to publication record in Ulster University Research Portal

Published in:

Unknown Host Publication

Publication Status:

Published (in print/issue): 10/11/2017

\section{Document Version}

Author Accepted version

\section{General rights}

Copyright for the publications made accessible via Ulster University's Research Portal is retained by the author(s) and / or other copyright owners and it is a condition of accessing these publications that users recognise and abide by the legal requirements associated with these rights.

\section{Take down policy}

The Research Portal is Ulster University's institutional repository that provides access to Ulster's research outputs. Every effort has been made to ensure that content in the Research Portal does not infringe any person's rights, or applicable UK laws. If you discover content in the Research Portal that you believe breaches copyright or violates any law, please contact pure-support@ulster.ac.uk. 


\title{
A Holistic Technology-Based Solution for Prevention and Management of Diabetic Foot Complications
}

\author{
Ian Cleland ${ }^{1}$, Joseph Rafferty ${ }^{1}$, Jonathan Synnott ${ }^{1}$, Jill Cundell ${ }^{2}$, Adele Boyd ${ }^{1}$, \\ Chris Nugent ${ }^{1}$, Priyanka Chaurasia ${ }^{1}$, Gareth Morrison ${ }^{3}$, Godfrey Madill ${ }^{4}$, Leeann \\ Monk- Ozgul ${ }^{5}$ and Stephen Burns ${ }^{6}$. \\ ${ }^{1}$ Computer Science Research Institute and School of Computing and Mathematics, Ulster \\ University, Newtownabbey, Co. Antrim, Northern Ireland, BT37 0QB. \\ \{i.cleland, j.rafferty, j.synnott, a.boyd, cd.nugent, p.chaurasia\}@ulster.ac.uk \\ ${ }^{2}$ Institute of Nursing and Health Research, Ulster University, Newtownabbey, Northern \\ Ireland, BT37 0QB. \{jh.cundell\}@ulster.ac.uk \\ ${ }^{3}$ Lava Group, 17 Falcon Road, Belfast, UK BT12 6RD g.morrison@thelavagroup.co.uk \\ ${ }^{4}$ Prosthetics Forum Northern Ireland, Belfast, Northern Ireland, BT9 7JB. \\ godfreymadill@gmail.com \\ ${ }^{5}$ Elemental Software, Holywell Trust, 10-12 Bishop Street, Derry, Northern Ireland, BT48 \\ 6PW. leeann@elementalsoftware.co \\ ${ }^{6}$ PillPacPlus, Unit 9, 20 Antrim Road, Ballymena, Northern Ireland, BT42 2BJ. \\ stephen@pillpacplus.com
}

\begin{abstract}
It has been estimated, on a global scale, that a limb is lost every 20 seconds due to diabetes. Effective treatment has, however, been shown to reduce the risk of amputations and foot ulcers. Self-management and the provision of effective foot care in the clinic and at home, is viewed as instrumental in providing appropriate care for people with diabetes. This in turn can reduce the costs associated with treating and managing diabetes and its associated complications. This paper presents a usability evaluation of a smartphone based solution for the management of diabetic foot disease. The solution combines novel thermal imaging with tailored educational content and gamification elements in an attempt to improve self-management of the condition. The solution was evaluated in a workshop setting by 7 participants. Overall, the participants felt the proposed solution would be a very worthwhile endeavor. They rated the usability and utility at an acceptable level (SUS 69.1/100). Notable suggested improvements focused on how educational content was formatted and searched in addition to the physical support provided to facilitate imaging of the feet.
\end{abstract}

Keywords: Self-management, diabetic foot disease, thermal imaging, m-health.

\section{Introduction}

By 2035 it is estimated that around 600 million people worldwide will have diabetes mellitus [1]. This increase threatens to bring huge financial instability to healthcare systems, including the National Health Services. The management of diabetes currently costs the National Health Service in the UK $£ 10$ billion each year [2]. If not properly 
managed and treated diabetes can lead to serious complications such as heart disease, stroke, blindness, kidney failure and amputation [1]. Among these complications, Diabetic Foot Disease (DFD), is one of the most debilitating, posing a substantial health and societal burden on patients and healthcare systems. Each year around 1 million people lose at least part of a leg as a consequence of DFD [3]. This equates to an amputation every 20 seconds worldwide [3]. People with diabetes are 15 times more likely to need an amputation than those without the condition [4]. Diabetic foot ulcers are the most prevalent problem, with a yearly incidence of around $2-4 \%$ in developed countries [1]. Typically, $25 \%$ of patients who present to a multi-disciplinary clinic with neuropathy will develop a foot ulcer. Of these foot ulcers, around $50 \%$ will become infected and $20 \%$ of these infected ulcers will lead to varying degrees of amputation [5]. New products and services that improve these statistics will have a major impact on the quality of life in addition to reducing the financial burden that DFD places on healthcare systems.

Self-management is viewed as instrumental in providing appropriate care for people with diabetes while, simultaneously, reducing the costs associated with treating and managing diabetes and its associated complications [6]. Self-management focuses on providing the individual with the skills and knowledge to stay healthy and prevent costly complications. For DFD, self-management activities may include, effective footcare, healthy eating, being physically active, monitoring of blood sugar, compliance with medications, good problem-solving skills, healthy coping skills and risk-reduction behaviors. Effective treatment reduces the risk of foot ulcers and amputations.

For prevention and care specific to DFD, it is recommended that patients check their feet daily [7]. Signs of foot ulceration may include: redness, pain, loss of sensation, callus or thickened skin around the ulcer. In addition to every day self-checks, the National Institute for Health and Care Excellence (NICE) recommends attending at least an annual foot review with a clinician to identify and treat ulceration quickly and effectively [7]. These checks aim to identify changes in foot health e.g. neuropathy, peripheral arterial disease or infection and advise the person of their risk status as per Diabetes UK's risk stratification. Nevertheless, a considerable number of people with diabetes are receiving inadequate care during annual foot checks, increasing their risk of amputation, according to research carried out by Diabetes UK [5]. In an online survey of almost 6,700 people with diabetes, approximately one third said they were not informed about their level of risk regarding foot problems during the check. The same proportion said they were not given advice about their foot care. [8] There is therefore a need to provide better education and tools for people with diabetes and primary care providers to provide adequate and effective foot care both at home and in the clinic.

This paper presents the design and usability evaluation of a self-management solution for the prevention and management of DFD. Specifically, the solution combines emerging thermal imaging technology for the identification of early signs of ulceration, with a smartphone application to deliver educational information pertinent to effective prevention and management of diabetic foot conditions. This is achieved through intelligent data analytics. The paper is structured as follows: firstly, a review of technology solutions for the management of DFD is presented. Following this an overview of the proposed solution for management and prevention along with the 
evaluation methodology is provided. The results of the usability evaluation are then presented and discussed.

\section{Background}

A patient's awareness of their risk and of the protective measures they can take is important in preventing diabetes-related foot ulceration, as has been evidenced in recent Cochrane database reviews $[9,10]$. Moreover, in its published Standards of Medical Care in Diabetes, the American Diabetes Association recommends self-monitoring of foot health and family involvement as useful strategies for improving diabetes healthcare outcomes [12]. A large element of this is the ability to carry out a thorough foot examination and identify signs of a foot infection.

In the UK, diabetes foot surveillance is mainly performed by the primary care health professionals and forms a part of their Quality and Outcomes Framework (QOF) programme [13]. Nevertheless, the 2010 National Health Survey diabetes-led patient survey showed that over $50 \%$ of people with diabetes claimed not to recollect having had their feet examined or being given any advice related to foot care [13]. This disparity suggests that an examination had either not been performed and/or the patients had not been engaged in the purpose and the findings of the foot examination. Home examinations, via a number of methods, have been proposed in order to thwart this disparity [12]. Various solutions exist that aim to detect DFD, nevertheless, the current in practice approach of manual inspection of the foot is still the most common method of detecting DFD. The following sections describe diagnostic tests and devices for identifying early signs of DFD.

\subsection{Devices for diagnosis}

Pressure perception, using a 10-g monofilament instrument, is the most commonly used screening test to identify loss of protective sensation in primary care as it is a relatively simple procedure and an abnormal result has been found to be associated with a 7.7fold increased ulceration risk [14]. It could potentially be used by non-professionals such as relatives, as it is quick to perform; however, it requires some training and supplying a device for each patient to be screened [12]. Pressure has been and remains to be an important indicator of ulceration $[5,6,7,8]$. In-shoe pressure, unfortunately, is a difficult and expensive parameter to measure. Pressure-sensing devices are available such as the Tekscan F-scan [15], however, these are expensive, have a relatively short product life span and are not be suitable for everyday use [16].

Temperature, is very closely associated with pressure and is considered as a more reliable measurement [17]. This is mainly due, to the availability of hardware with thermistors providing highly accurate measurement in a small form. Less attention has, however, been directed towards foot plantar temperatures as an indicator of ulcer risk as compared to pressure [17]. Studies have shown that there is a relationship between increased temperature and foot complications in diabetes [17]. Increased temperature may be present up to a week before a foot ulcer occurs. In such an early stage of the disease, patients seldom feel pain because of neuropathic sensory loss, indicating that 
increased temperature can be a useful predictive sign of foot ulceration and sub-clinical inflammation of the feet. Infrared (IR) thermometers are commonly used to assess temperature differences in the feet caused by DFD [17].

The use of handheld dermal IR thermometers in the home environment has been validated by randomized controlled trials for the prevention of recurrent diabetic foot ulceration. Three randomized controlled trials tested patient monitoring of foot skin temperature as an indicator of an impending ulcer [18-20]. These studies found a significant reduction in new foot ulcers with use of a temperature monitoring device. This technology, however, requires, the temperature to be measured manually on specific spots on the foot. This makes it subjective, and it is difficult to obtain the temperature distribution of the whole foot. Furthermore, this technology misses the opportunity for automatic detection of DFD [21].

Another method, which is less ambulatory, is the use of liquid crystal thermography in such products as Thermoscale, Tempstat and SpectraSole Pro 1000 [23]. TempStat uses specialized liquid crystal technology (LCT) to monitor the temperature on the bottom of the feet [23]. TempStat has been approved by the U.S. Food and Drug Administration to identify areas of inflammation. Some limitations to LCT include low sensitivity, low image resolution and pressure sensitivity, in addition to the disadvantage of a very demanding device handling during contact of the crystals with examined body area [21]. Compared with LCT, IR camera systems have the advantage of being noncontact, which prevents unwanted pressures and the transmission of pathological organisms. The temperatures of noncontact foot regions, such as the medial arch, can be easily measured with IR camera systems. Additionally, it is capable of measuring the dorsal side of the foot as well. As such, IR camera systems show a greater potential for tele-medical applications [21]. Moreover, the authors have provided evidence that detection of temperature differences are independent of the quality of the IR camera used and that it can be achieved without image processing algorithms [23]. Nevertheless, LCT offers the possibility of both static and dynamic measurements on the plantar aspect of the foot [23]. It was concluded that the IR camera is the most usable and cost-effective thermography option for the prevention of diabetic foot complications [23]. They also proposed that the use of such devices over the next 5 years in a patient centered fashion may not only aid in the early detection and avoidance of foot complications, however, also contribute to patient education, encouraging a healthier lifestyle [23]. A number of companies supply IR cameras for thermography, however, to date no products targeted specifically at the DFD market are commercially available.

\subsection{The Role of Education}

In addition to daily foot examination, education has an important part to play in the prevention of DFD. Lack of education leads to unawareness of diabetic foot problems and their prevention. Interestingly, one study demonstrated that $90 \%$ of screened diabetic patients had poor knowledge about their disease and $96.3 \%$ had poor awareness about its control [18]. Education has been recognized as a fundamental aspect of diabetes treatment for all age groups [24-26]. This is particularly the case for older patients, however, it has been suggested that diabetic education would be most effective 
if it is tailored to the particular needs of older learners [24]. This includes individualized self-management plans and advice, and should also consider the particular health characteristics of older adults. Educational provision should be adaptable to conditions such as visual and hearing impairments and cognitive and physical abilities [24, 26]. Several apps are now available for smartphones which provide stratified educational content. Recently the College of Podiatry, in collaboration with the special advisory group Foot in Diabetes UK and the British Association of Prosthetists and Orthotists, have created two apps to enable increased knowledge around the problems that can occur for those with DFD. One is for health care professionals who undertake diabetic foot screenings, the other is for people with diabetes to ensure they are empowered to prevent problems with their feet. These apps provide risk categorization to groups of high, moderate and low risk and provide relevant information and advice on self-care and assessment. Whilst these apps provide excellent educational resources tailored to the user, they do not include tools to aid in assessment of feet at home as previously detailed.

Whilst more contemporary methods, such as pressure/temperature insoles, do provide the flexibility to measure continuously, many insoles are currently cumbersome to use, having external power and processing units and users must remember to charge them and transfer them from shoe to shoe. Also, the data generated is not intuitive to interpret and can require clinical knowledge to do so. Furthermore, none of the solutions currently available provide educational information, which is an important part of any self-management programme. The solution developed and evaluated within this work, seeks to fill these gaps in the market by providing a non-intrusive and automatic method of detecting DFD. The project seeks to utilize thermal imaging technology to implement an ambulatory, non-invasive method of automatically detecting early signs of DFD and providing the user with the knowledge to effectively address these issues. Additionally, the solution will tackle prevention of DFD though promotion of healthy lifestyle, education and prompting the individual to check their feet regularly. Such monitoring and education, if more widespread and frequent, can potentially reduce amputation rates and thus reduce associated costs attributed to diabetic related amputation. The following Sections provide an overview of the proposed solution.

\section{Solution to prevent and manage DFD}

The developed solution has two main components, a mobile application and an associated thermal camera, and an analysis platform - a cloud-based service that hosts a web interface. This thermal camera has been validated within previous research for the purposes of hotspot detection (temperature difference of $>2^{\circ} \mathrm{C}$ is defined as a hotspot) [27]. The mobile app captures thermal vision data and raw RGB images. These are then uploaded, though a service, to the backend database. The backend stores this data in database and processes it to extract metrics to automatically identify hotspots. The Web-API of the local login scenario uses the standardized Open Authentication (OAuth) mechanism to authenticate the request and to manage data flow according to authorization credentials. The architecture of the developed solution is presented in Figure 1. Thermal images are captured by the thermal sensor and the associated mobile 
app. These images are processed locally for hotspot detection before being uploaded to the backend via a rest endpoint. These images are combined with a personalized profile and patient history information to generate a personalized profile for tracking and processing the thermal images. This profile is generated through the application of machine learning techniques. A Web User Interface (UI) allows professional caregivers to view images and update patient profiles and educational content.

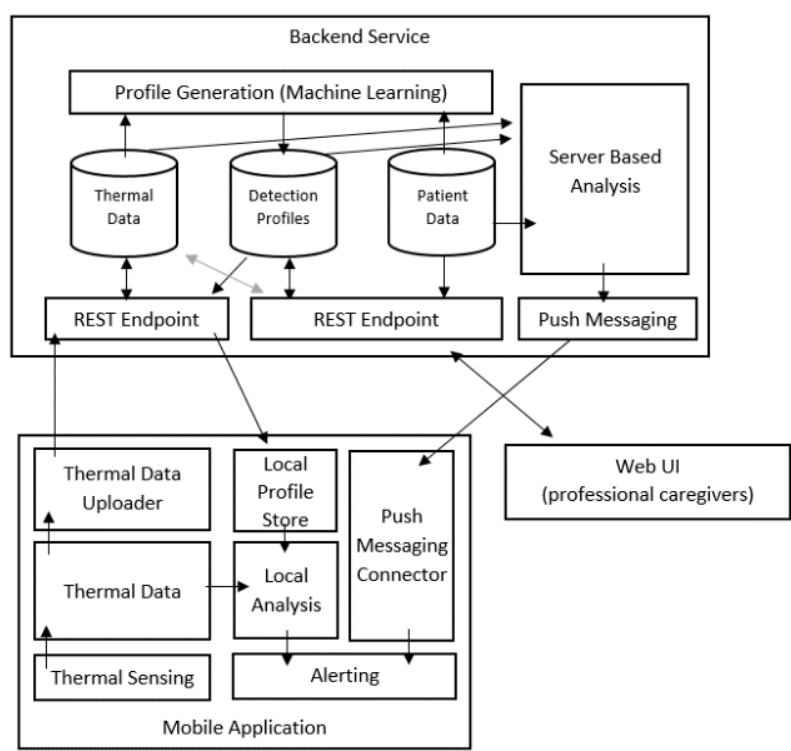

Fig. 1. Overview of the thermal imaging architecture developed for the management of DFD.

The educational app, delivers tailored educational facts and suggestions to aid in selfcare of DFD. Educational content is delivered in two modes. The first is a short fact and suggestion pair that is pushed to the user on a daily basis. These daily facts are short, evidence backed, statements with which a user can leverage to manage, prevent or reduce their risk of developing DFD. Educational content is also available through educational resources/articles, which can be multimodal, containing text, images or video content. These articles relate to self-management, prevention and care. Educational content is stratified based on risk category in a similar fashion to other applications. Both the educational resources and daily facts can be managed and populated through a content management system. This allows non-technical users to update and amend educational content based on clinical or research insight. In addition to the delivery of educational content and capture/review of thermal imaging, the mobile app also allows the user to rate educational content and update user profile information. An overview of the educational app is provided in Figure 2. 


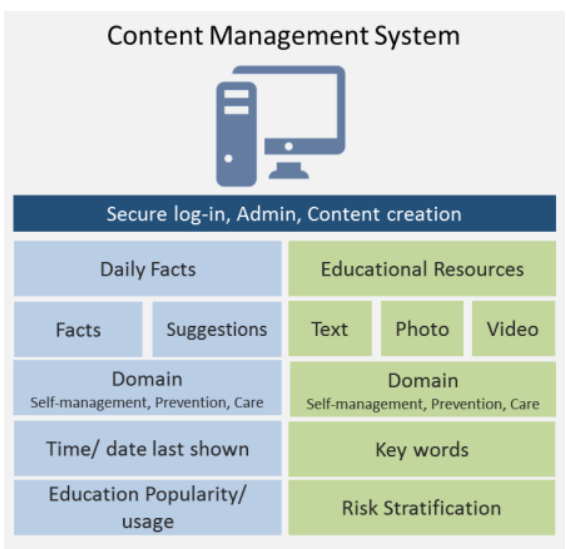

(a)

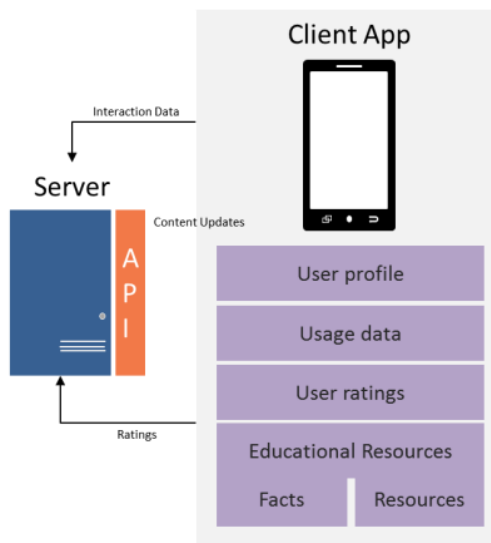

(b)

Fig. 2. Overview of educational client app and content management system. The client app (a) allows for interaction with the user to view and rate educational content. The content management system (b) provides the ability for a non-technical user to create and tailor educational content and facts.

In addition to the thermal imaging analysis and educational components of the app, a number of gamification elements have been included. The aim of these is to motivate the user to sustain engagement with the solution and develop self-management habits through behavior change. Figure 3 below shows the interface for the mobile app.

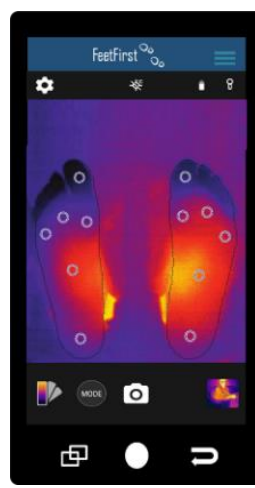

(a)

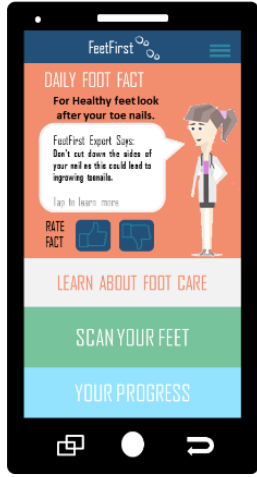

(b)

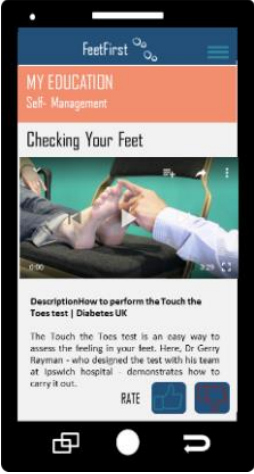

(c)

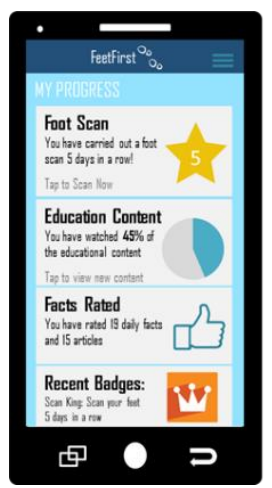

(d)

Fig. 3. Screenshots illustrating the various functions of the app including a) thermal scanning of feet, b) daily facts, c) educational resources and d) gamification.

\section{Usability Evaluation}

This Section provides details of the usability evaluation undertaken in order to assess whether the proposed solution would be usable by a representative cohort and to identify any improvements which could be made in order improve usability and support use of the solution independently. 


\subsection{Methodology}

To evaluate the solution a usability workshop was held with members of the Diabetes UK voluntary group. Seven participants (3 female, 4 male), aged between 30 and 67 took part in the evaluation. This convenience sample was used simply to evaluate the usability of the system. Previous research into usability assessments has shown that around $80 \%$ of the errors in an interface can be uncovered using just 5 participants [28, 29] A varied age group from this representative sample is considered, to capture as many view points as possible. Participants reported to be in good general health, with only one reporting issues with mobility and dexterity. Only one of the participants had a history of previous diabetic foot infection. All users owned a smartphone (5/7 Android, 2/7 iOS) and reported to be familiar with smartphone apps.

After a brief introduction to the project and an overview of the solution, participants were invited to undertake two tasks that showcased the features of the app thus enabling users to evaluate usability. Tasks where split into two components, as presented in Table 1. Task 1 focused on the usability and gamification aspects and asked users to review both how the content was presented in addition to the literacy of the content itself. Task 2 asked participants to review instructions of how to use the thermal camera and then to take an image of their feet. Participants were asked to scan one foot at a time and then both feet simultaneously, if able to.

Table 1. Task list completed by workshop participants in order to showcase functionality of the app.

\begin{tabular}{|c|c|c|}
\hline & Task Description & Task Purpose \\
\hline 1 & $\begin{array}{l}\text { Complete the following steps } \\
\text { - Launch app on mobile phone device } \\
\text { Review the home screen and identify what options are } \\
\text { available. } \\
\text { - Rate the daily fact on the home screen. } \\
\text { Navigate to the educational content and review each of the } \\
\text { available information sources under self-management. } \\
\text { - Navigate to the "Your Progress" section of the app and } \\
\text { review the recent badges. } \\
\text { - Tap to find out more information about the available badges. } \\
\text { Access the app menu and navigate back to the app home } \\
\text { screen. }\end{array}$ & $\begin{array}{l}\text { To demonstrate overall } \\
\text { function of app and } \\
\text { highlight specific } \\
\text { features and to obtain } \\
\text { user opinion on } \\
\text { usefulness of features } \\
\text { and to obtain service } \\
\text { user opinion on } \\
\text { accessibility of } \\
\text { educational materials }\end{array}$ \\
\hline 2 & $\begin{array}{l}\text { - Access the "Scan your feet" section and use the "show me } \\
\text { how" instructions to find out how to attach the camera to the } \\
\text { mobile device. } \\
\text { - Turn on the thermal camera } \\
\text { - Use the app to capture a thermal image. } \\
\text { - Use the Thermal camera to try to capture an image of one } \\
\text { foot at a time. } \\
\text { Use the thermal camera to try and capture an image of both } \\
\text { feet simultaneously. }\end{array}$ & $\begin{array}{l}\text { To assess the practical } \\
\text { issues around using the } \\
\text { camera to capture } \\
\text { thermal images of the } \\
\text { feet. }\end{array}$ \\
\hline
\end{tabular}

Following completion of the tasks, participants were asked to complete an evaluation questionnaire. This questionnaire had 3 sections; 1) usability of the app and 2) standard System Usability Scale (SUS) and 3) usage of the thermal camera and scanning of the feet. The questionnaire asked them to rate various aspects about the 
app including; look and feel, ease of use, ease of navigation and the utility of the app. Participants were also asked about how easy it would be to learn to use the app and how long that learning would take, how easy it was to use and attach the camera and how easy it was to scan both and/or one foot independently. Finally, participants were also asked to provide comments on what they felt was good or bad about the app and any improvements they would recommend. In addition to these customized questions about the app, the usability was measured using the System Usability Scale (SUS) [30]. This validated metric provides a reliable tool for measuring usability. It consists of a 10-item questionnaire with five response items; from Strongly Agree to Strongly Disagree. The SUS is scored out of 100 with an acceptable score being greater or equal to 70 [31]. In addition to the usability of the app, participants were also asked to comment on the setup process for the camera and the practical issues of imaging one's feet. Results from the usability assessment are presented in the following Section.

\section{Results}

In terms of the smartphone application, participants reported that it was generally very easy to use and thought the concept of combining thermal imaging and education content 'very worthwhile'. In general participants found the app responsive (Mean$3.57 / 5$ ) easy to use (Mean- 3.57/5) and easy to navigate (Mean- 3.57/5). Participants also felt that it would be easy (Mean- 3.7/5) and quick to learn to use the app with most users stating they could do so within a few hours (5 reported Hours, 2 reported Minutes). When asked to consider how useful the app had been in improving their knowledge of diabetic foot conditions, users reported very useful (Mean- 4.14/5). Full results are reported in Figure 4.

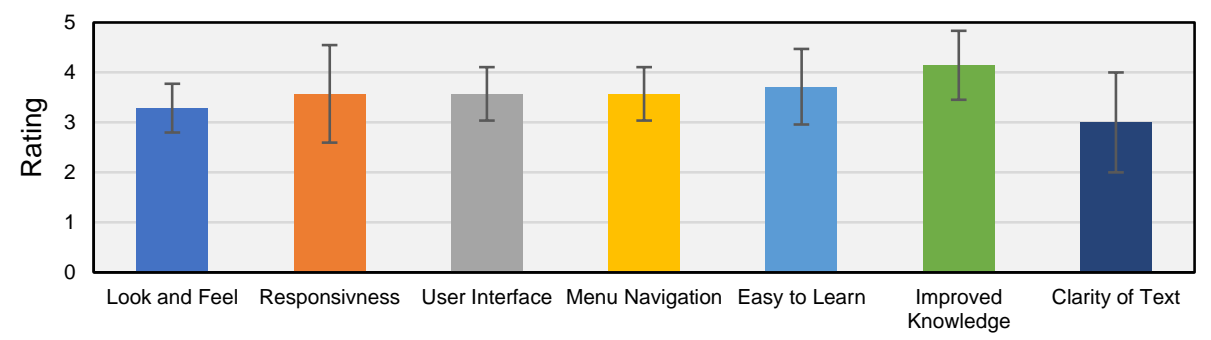

Fig. 4. General usability results from the questionaire for app look and feel, responsivness, user interface design, menu navigation, ease of learning, ability to improve knoweledge and clarity of text. Error bars represent standard deviation.

The SUS scores echoed the same results as the general usability questions. Results for the SUS are represented with a single score ranging between 0-100. Scores below 50 indicate poor design; scores between 50 and 70 indicate an acceptable design; scores between 70 and 85 indicate a good design; and scores above 85 indicate an excellent design [31]. Over all the app and thermal imager received an SUS score of 69.1/ 100, placing it in the acceptable range of design, however, a number of changes could be 
made to improve this, as presented in the discussion Section. Average scores with standard deviation for each question in the SUS are presented in Figure 5.

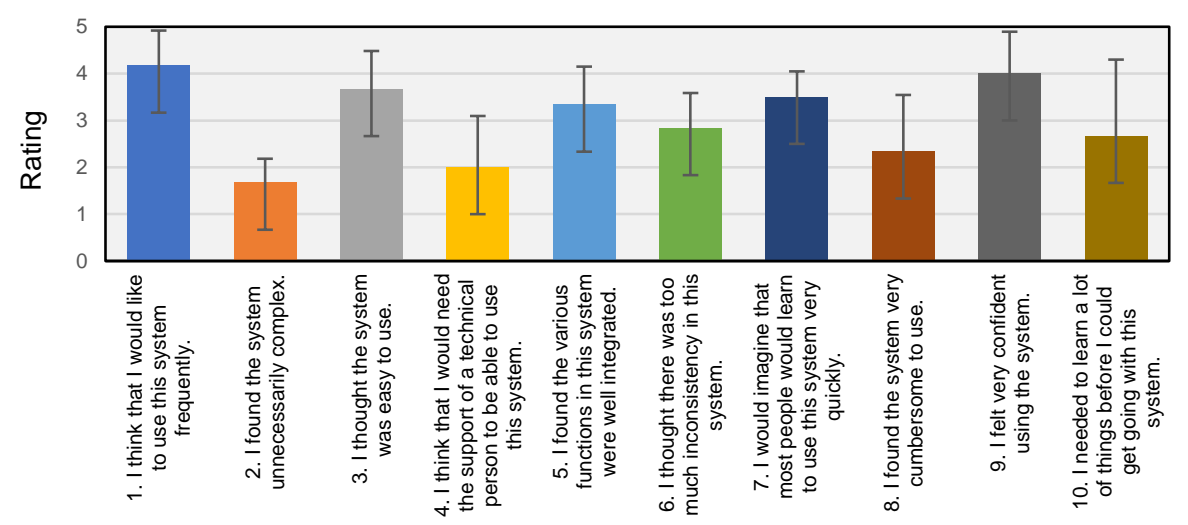

Fig. 5. Average rating given to each question in the System Usability Scale (SUS). Error bars represent standard deviation. Each question was rated on a scale of 1 to 5 with 1 being strongly disagree and 5 being strongly agree.

When considering the use of the thermal camera, participants reported that the camera was simple to use (Mean 4.42/5). Participants also reported that the instructions provided showing how to use the app/camera, turn on the camera and launch the app were clear and easy to understand (Mean 4.85/5). When asked to consider how easy it was to capture an image of both feet simultaneously, only 3 of 7 participants could do so, and provided a rating of 2.67/5. The other participants were unable to complete the task. Six of the 7 participants could scan one foot at a time, with one participant citing poor flexibility as a limiting factor. Participants rated scanning one foot as 3.83/5 showing that whilst possible, some help would be required to facilitate this on a daily basis. Participants noted that capturing an image of the feet may be easier if they had assistance from a carer or family member. All the participants agreed that they would be comfortable to ask a family member to scan their feet on a regular basis, though one participant highlighted the increase in carer burden associated with this.

When considering how the app could be improved, participants commented on the vast amount of text in the educational content. This content was repurposed from wedbased educational resources and therefore contained large bodies of text. Participants commented that this could be improved by summarizing the main points and providing more images. They also mentioned the need for better searching capabilities within the app to find content that they were looking for. This included key word searching and bookmarking sections so that text could be skipped and move to the section of interest. A similar comment was mentioned for video content, where participants wished to skip to sections of the video that they were interested in without watching the whole video. This could be further improved by stratifying the educational content based on the user's risk level and their health literacy.

In general participants felt the solution functioned well and would be a useful tool in managing their feet and avoiding complications. Participants felt the solution would need to be fully embraced by clinicians to be adopted fully into the foot care pathway. 


\section{Conclusion}

This paper describes a holistic solution for the prevention and management of DFD. The solution combines a thermal imaging solution with tailored educational content to provide the tools and knowledge required for a user to better manage and prevent deterioration of their condition. In an initial usability evaluation, the solution has been assessed in a workshop setting by 7 members of a representative cohort. General usability ratings and scores from the SUS have proven promising, however, there is room for improvement, particularly in terms of how educational content is delivered and the support necessary to capture clear and reliable images of the participant's feet. Additionally, the workshop has highlighted the need to engage with clinicians to have the solution adopted into the care pathway. To assess whether this solution is effective in improving foot care and capturing early signs of DFD, the solution will need to be evaluated longitudinally. Future work will therefore aim to evaluate the solution longitudinally in a home setting to assess its impact on prevention, self-care and knowledge of diabetic foot complications. Additionally, we will seek to engage with clinicians to gain insight into how this solution could be integrated further within a foot care pathway.

\section{Acknowledgment}

Invest Northern Ireland is acknowledged for supporting this project under the Competence Centre Programme Grant RD0513853 - Connected Health Innovation Center. The authors would like to thank Diabetes UK for organising the workshop and the participants who attended and provided valuable feedback.

\section{References}

1. Bakker, K., Apelqvist, J., Lipsky, B. A., Van Netten, J. J., \& Schaper, N. C. "The 2015 IWGDF guidance documents on prevention and management of foot problems in diabetes: development of an evidence-based global consensus." Diabetes/metabolism research and reviews 32.S1 (2016): 2-6.

2. Health and Social Care Information Centre. National diabetes audit-2011-12. http://www.hscic.gov.uk/catalogue/PUB12421. (accessed March 12)

3. Bakker, K., and N. C. Schaper. "The development of global consensus guidelines on the management and prevention of the diabetic foot 2011." Diabetes/metabolism research and reviews 28.S1 (2012): 116-118.

4. Diabetes UK. "More than 135 diabetes amputations every week." https://www.diabetes.org.uk/About_us/News/More-than-135-diabetes-amputations-every-week/ (Accessed 16th September 2015).

5. Putting feet first: commissioning specialist services for the management and prevention of diabetic foot disease in hospitals. Diabetes UK, 2009.

6. $\quad$ Ellis, Pete, and Claire Senior. "Diabetes education: the big missed opportunity in diabetes care." Diabetes UK (2015).

7. NICE clinical guideline (CG19) Diabetic foot problems: prevention and management (2016).

8. Diabetes UK online footcare survey (https://www.diabetes.org.uk/About_us/News/Poordiabetes-foot-checks/Footcare-survey-results/) (2014) (Accessed September 2015). 
9. Schillinger, D., Grumbach, K., Piette, J., Wang, F., Osmond, D., Daher, C., ... \& Bindman, A. B. "Association of health literacy with diabetes outcomes." Jama 288.4 (2002): 475-482.

10. Duke SA, Colagiuri S, Colagiuri R. Individual patient education for people with type 2 diabetes mellitus. Cochrane Database Syst Rev 2009; 1: CD005268.

11. Dorresteijn JA, Kriegsman DM, Assendelft WJ, Valk GD. Patient education for preventing diabetic foot ulceration. Cochrane Database Syst Rev 2010; 5: CD001488.

12. American Diabetes Association. Standards of Medical Care in Diabetes-2013. Diabetes Care 2013; 36: S11-S66.

13. Sharma, S., C. Kerry, H. Atkins, and G. Rayman. "The Ipswich Touch Test: a simple and novel method to screen patients with diabetes at home for increased risk of foot ulceration." Diabetic Medicine 31.9 (2014): 1100-1103.

14. Square, Trevelyan, and Boar Lane. "Patient Experience of Diabetes Services." (2013).

15. Lung, C. W., Hsiao-Wecksler, E. T., Burns, S., Lin, F., \& Jan, Y. K. "Quantifying Dynamic changes in Plantar Pressure gradient in Diabetics with Peripheral neuropathy." Frontiers in Bioengineering and Biotechnology 4 (2016).

16. Taborri, J., Palermo, E., Rossi, S., \& Cappa, P. "Gait partitioning methods: a systematic review." Sensors 16.1 (2016): 66.

17. Bus, S. A., Netten, J. J., Lavery, L. A., Monteiro-Soares, M., Rasmussen, A., Jubiz, Y., \& Price, P. E. "IWGDF guidance on the prevention of foot ulcers in at-risk patients with diabetes." Diabetes/metabolism research and reviews 32.S1 (2016): 16-24.

18. Liu, C., van Netten, J. J., Van Baal, J. G., Bus, S. A., \& van Der Heijden, F. "Automatic detection of diabetic foot complications with infrared thermography by asymmetric analysis." Journal of biomedical optics20.2 (2015): 026003-026003.

19. Lavery L.A, Higgins KR, Lanctot DR, Constantinides GP, Zamorano RG, Athanasiou KA, et al. Preventing Diabetic Foot Ulcer Recurrence in High-Risk Patients Use of temperature monitoring as a self-assessment tool. Diabetes Care. 2007;30: 14-20.

20. Armstrong DG, Holtz-Neiderer K, Wendel C, Mohler MJ, Kimbriel HR, Lavery LA. Skin temperature monitoring reduces the risk for diabetic foot ulceration in high-risk patients. Am J Med. 2007; 120:1042.

21. Jung, Y, et al. "Ground reaction force estimation using an insole-type pressure mat and joint kinematics during walking." Journal of biomechanics47.11 (2014): 2693-2699.

22. Lavery LA, Higgins KR, Lanctot DR, Constantinides GP, Zamorano RG, et al. Home monitoring of foot skin temperatures to prevent ulceration. Diabetes Care. 2004;27:2642-7.

23. Frykberg, R. G., A. Tallis, and E. Tierney. "Diabetic foot self examination with the Tempstat as an integral component of a comprehensive prevention program." The Journal of Diabetic Foot Complications 1.1 (2009): 13-18.

24. Quinn, C.W, Raymond Bond, and Chris Nugent. "Ontological modelling and rule-based reasoning for the provision of personalized patient education. "Expert Systems (2015).

25. International Diabetes Federation (2013b) Managing older people with type 2 diabetes global guideline,http://www.idf.org/guidelines/managing-older-people-type-2-diabetes [Accessed 24 November 2015].

26. Mcdowell, J., F. Brown and D. Matthews (Eds)(2007) Diabetes A Handbook for the Primary Healthcare Team, 2nd edn. Edinburgh: Churchill Livingstone.

27. Rafferty J., Cleland I., Nugent C., Armstrong, K. and Madill G., (2016) An evaluation of contactless thermal sensing elements for use in a technology based diabetic foot disease detection solution. In: IEEE Engineering in Medicine and Biology Society Conference, Orlando, Florida, US., pp. 4379-4382. IEEE, EMBC'16.

28. Nielsen, J. Estimating the number of subjects needed for a thinking aloud test. International Journal of Human-Computer Studies 41, (1994), 385-397. 9.

29. Nielsen, J. and Molich, R. HE of user interface. In CHI ' 90 Conference Proceedings. ACM, 1990, 249-256.

30. Brooke, John. "SUS-A quick and dirty usability scale." Usability evaluation in industry 189.194 (1996): 4-7.

31. Bangor, Aaron, Philip Kortum, and James Miller. "Determining what individual SUS scores mean: Adding an adjective rating scale." Journal of usability studies 4.3 (2009): 114-123. 